\title{
Ultrasound image segmentation through deep learning based improvised U-Net
}

\author{
Nayana R Shenoy ${ }^{1}$, Anand Jatti ${ }^{2}$ \\ ${ }^{1}$ Dr. AIT, Bangalore, India \\ ${ }^{2} \mathrm{RVCE}$, Bangalore, India
}

\begin{tabular}{l} 
Article Info \\
\hline Article history: \\
Received May 7, 2020 \\
Revised Aug 7, 2020 \\
Accepted Oct 1, 2020 \\
\hline
\end{tabular}

Keywords:

Improvised U-Nets

Thyroid gland

Thyroid segmentation

\begin{abstract}
Thyroid nodule are fluid or solid lump that are formed within human's gland and most thyroid nodule doesn't show any symptom or any sign; moreover there are certain percentage of thyroid gland are cancerous and which could lead human into critical situation up to death. Hence, it is one of the important type of cancer and also it is important for detection of cancer. Ultrasound imaging is widely popular and frequently used tool for diagnosing thyroid cancer, however considering the wide application in clinical area such estimating size, shape and position of thyroid cancer. Further, it is important to design automatic and absolute segmentation for better detection and efficient diagnosis based on US-image. Segmentation of thyroid gland from the ultrasound image is quiet challenging task due to inhomogeneous structure and similar existence of intestine. Thyroid nodule can appear anywhere and have any kind of contrast, shape and size, hence segmentation process needs to designed carefully; several researcher have worked in designing the segmentation mechanism, however most of them were either semi-automatic or lack with performance metric, however it was suggested that U-Net possesses great accuracy. Hence, in this paper, we proposed improvised U-Net which focuses on shortcoming of U-Net, the main aim of this research work is to find the probable Region of interest and segment further. Furthermore, we develop High level and low-level feature map to avoid the low-resolution problem and information; later we develop dropout layer for further optimization. Moreover proposed model is evaluated considering the important metrics such as accuracy, Dice Coefficient, AUC, F1-measure and true positive; our proposed model performs better than the existing model.
\end{abstract}

This is an open access article under the CC BY-SA license.

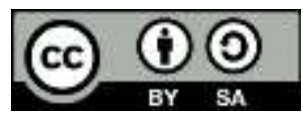

Corresponding Author:

Nayana R Shenoy, Anand Jatti

Dr. AIT, Bangalore, India

Email : @ nayanashenoy99, @ rediffmail.com

\section{INTRODUCTION}

Human body comprises several endocrine organ, thyroid is one of the essential endocrine organ that lies in the near the neck below the cartilage, thyroid hormone regulates the metabolism of human body. Further, some of the thyroid nodules are of proper margins, some of them are irregular in shape. In general, thyroid nodules are categorized into three distinctive form i.e. crystic, solid and integration of both crystic and solid nodules, also sometimes classification of thyroid is known as isoechoic, hypopechoic or hyperechoic. Moreover in last decade it is observed that thyroid cancer has been increasing with rate of $4.5 \%$ [1], and through the research it was found that united states have reported more than fifty thousand cases of thyroid cancer last year and more than two thousand people have died due to the cancer [2,3]. In general 
thyroid nodules are benign with 4.5-6\% of malignancy, hence researcher and radiologist recommends screening process.

Moreover, Ultrasonography is of the important and popular tool to evaluate the thyroid nodule. Ultrasonography is mainly used due to its cost effective and high sensitivity occurrence. However, it requires clinical experience of radiologists to identify the thyroid cancer since the radiologists performs the diagnosis based on the nodule characteristics and this makes very much challenging for thyroid cancer detection. Hence, several researcher work on computer added design aka CAD to automatically classify the thyroid nodules. In general CAD comprises three component i.e. detection of nodule, image feature extraction and classification. Detection of nodule is carried through process known as segmentation, segmentation is the process of finding probable ROI (region of interest). Moreover, deep learning methodology provides the improvisation in image processing, Figure 1 shows the general process of segmentation. Further, it is divided into five blocks, first block shows ultrasound image in second block the region is identified later in third block model is trained using the any deep learning methodology. Once the model is trained then it is segmented and the output is given, several methodologies [4-7].

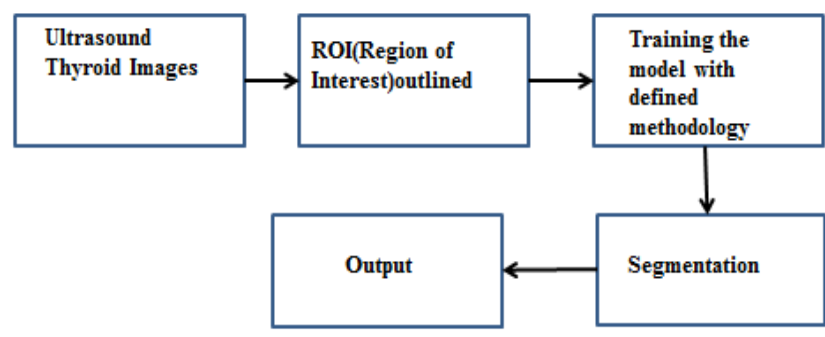

Figure 1. General procedure for segmentation

Segmentation is one of the important role for detecting the nodules and generating the ROI (region of interest) in thyroid ultrasound images; moreover absolute segmentation of thyroid nodule helps in achieving better performance in CAD model. However, segmentation is one of the challenging task as manual segmentation are time consuming and differs from huge variability. Some of the researcher have proposed semi-automatic segmentation for ultrasound image, however it solves only the partial problem as it still requires human interaction and this further causes in widespread application of CAD model in thyroid US-image [8]. Driven by the clinical requirement and demand in application it becomes top most priority to develop the full automatic method; further researcher have focused on active contour based mechanism to be applied on either level set form or parametric form [9]. Although active contour helps in finding the probable region, it is totally dependent on pre-processing and initialization, this possesses high error rate of learning if pre-processing fails. Other researcher have also developed conventional learning method, however these worked in parts and fail to achieve absolute segmentation since most of the method are based on the handcrafted features. Extracting the feature is another complicated task which requires selecting feature and integrating feature through classifier.

In any image processing technique segmentation plays an important role as inaccurate segmentation might lead to misdiagnoses, especially which are boundary based. Moreover accurate segmentation is considered as the basis of difference between malignant and benign, further thyroid gland segmentation is basic need for estimation of thyroid volume. Thyroid volume is used for analyzing the secretion of thyroid hormone, this needs to be considered as priority as it helps in finding the thyroid abnormality. However clinically it is highly improbable for estimating these characteristics through humans. Hence, segmentation is very important in gland segmentation and nodule segmentation. Moreover Ultrasound Image segmentation has been part of research from past few decades and most of them were machine learning based as machine learning performs better than any traditional model, However machine learning method does face some serious issue such as these method requires adequate amount of marked dataset and it takes lot of time to train the model. Considering these facts we have developed a segmentation model to find the region of interest. The contribution of this research are highlighted through the below points.

a) At first we address issue of conventional U-Net architecture, these issue includes the problem of low image resolution, high resolution and optimization in number of pooling

b) We propose an improvised U-net for thyroid cancer image segmentation, the main aim of proposed model is to find the ROI. 
c) Improvised U net focuses on achieving the High level feature map and low level feature map to avoid the resolution problem occurred in basic U-Net architecture.

d) Further in order to achieve the better performance optimization is carried out through dropout layer

e) Proposed model is evaluated considering standard dataset.

f) Further Improvised U-Net is evaluated considering performance metric such as Dice Coefficient and truth positive rate.

This research work has been designed in a standard work, as first section involves background about thyroid, thyroid cancer and its various aspect, further in the same section we highlight the shortcoming of some of the existing segmentation model. Further, in same section we discuss motivation and contribution of this research work. Second section discuss the overall Improvised U-net model along with its mathematical formulation, Improvised U-Net is evaluated in third section considering various performance metrics.

\section{RELATED WORK}

According to different segmentation targets, the studies of thyroid ultrasound image segmentation are divided into two directions, namely, thyroid gland segmentation and thyroid nodule segmentation. Although the targets of the two segmentation applications are different, the method categorizations of the two segmentation applications are roughly the same. In [10], compared all the three segmentation method which includes LRAC (localized region) [11], ACWE and DRLSE (distance regularized) [12]; LRAC used local parameter for constructing energy function for segmentation, in here background and foreground considering the small local areas; here each point is considered in separate way for optimizing the local energy. DRLSE helps in utilizing the external energy and distance regularization term for achieving the desired edges through contour; further they implied the these three model to segment the thyroid ultrasound images. Further, it is observed that LRAC observed the shortest iteration with better accuracy, however they did not give the evaluation results in case of thyroid gland in US-image; similar case was observed in [13]. Few researcher used graph cut [14] for segmenting the thyroid US-image, graph cut is popular and important optimization algorithm which associate the segmentation problem with the min-cut problem; however this method was based on semi-automatic method and further it requires manual sketch of background and foreground in USimage. Moreover, graph cut model is used for segmenting the 1416 2D images and dce coefficient as considered for evaluation and achieves 76.5\%. In [15], developed an automatic segmentation method for multiple organs, which was mainly based on echogenicity similarity, however it failed miserably due to its accuracy issue. In recent some of the method like [16] developed U-net based CNN and achieves the dice coefficient of 0.876, it was better than their traditional approach, further [17] which was another U-net based $\mathrm{CNN}$ and achieves poor results of dice coefficient value of 0.538 considering thyroid gland. They used radial based-NN and patch based classification is used for training and achieves the accuracy of 96.52, however the result was mainly based on selected images. In [18], developed another automatic method which showed that manual tracing is better for segmentation and does provide the better understanding of methodology due to tracing, however it requires highly experienced radiologists [19, 20] developed an automatic method for segmenting the thyroid glands in 2D images, this method was named on RBFNN. Further 3D thyroid volumes were estimated based on 2D image. Here segmentation is considered in four step i.e. Image enhancement and probable region identification, extracting features and training RBFNN along with thyroid recovery. Moreover Image processing was carried out to speckle noise and enhance the ultrasound image and hence reduce the computation amount. Furthermore, in few methods 2D thyroid ultrasound image, vertical projection is carried out to for locating the probable region. It used AWMF and morphological operation is proposed for speckle noise reduction, further gray level compensation is used for contrast adjusting between the thyroid and background. They applied six texture feature which includes mean, variance, coefficient, histogram and block difference and intensity difference. RBFNN comprises three layers namely input layer, hidden layer and output layer [21] adopted the segmentation of thyroid gland based on the machine learning methods and support vector machine, here the whole segmentation was parted into two distinctive parts i.e. thyroid gland segmentation and nodule recognition. Thyroid gland segmentation contains three parts i.e. image enhancement, speckle noise and increasing the image quality [22]. Proposed FNN for segmentation purpose, here nine different features were considered which includes the thyroid gland region, histogram energy, histogram kurtosis, histogram skewness, histogram entropy, histogram variance standard deviation, mean, HAARV and HAARM, their accuracy was better, however they considered only limited set of images. In [23], developed a method which was trained with supervised learning algorithm for constructing the classifier, the main drawback was that it was tested on dataset of 5 images [24]. Proposed U-net model to segment the whole segment considering encoder and decoder, here encoder is responsible classifying and identifying the ultrasound image pixel and decoder was used for finding the specific part of the location. Here 
to avoid the overfitting data augmentation is carried out, this model considers the fair amount of data for model evaluation and achieves better than the other model.

\section{PROPOSED METHODOLOGY}

Segmentation is one of the primary steps in identification of thyroid cancer, further U-Net has gained popularity due to its capability of analysing the biomedical image and promises better efficiency. However, conventional U-Net architecture has several drawbacks. Main drawback is low-resolution image information in designed feature maps, this directly effects the efficiency of the model. Other drawback is it is very difficult to optimize the pooling operations, hence to avoid such issue we developed Improvised U-net where apart from the conventional U-net two feature map are designed namely high level and low level which helps in avoiding the low resolution issue. In here at first we design the U Net which is biomedical architecture of convolution neural network, later two feature maps are designed.

\subsection{Preliminaries}

U-net has Convolution layer, which comprises two operations i.e. Convolution and activation along with pooling; convolution layer reduces the computational complexities through distributing kernel coefficient for each feature map. Further, it generates various features in spatial domain and each layer has several kernels. Moreover, output of convolution layer and pooling in fully connected layer is computed in fully connected network. Fully connected network is given in the below,

$$
e^{k}=\left\{e_{1}^{k}, e_{2}^{k}, \ldots \ldots, e_{m_{k}}^{k}\right\}
$$

Similarly kernel is given as,

$$
y^{k}=\left\{y_{1}^{k}, y_{2}^{k}, \ldots \ldots, y_{m_{k}}^{k}\right\}
$$

And bias value is given as,

$$
\mathcal{D}^{k}=\left\{\mathcal{D}_{1}^{k}, \mathcal{D}_{2}^{k}, \ldots \ldots, \mathcal{D}_{m_{k}}^{k}\right\}
$$

Activation in the kth layer is given in the below.

$$
\begin{aligned}
& e_{l}^{k}=h\left(\mathcal{D}_{l}^{k}+\mathcal{Y}_{l}^{k} \otimes \mathcal{F}\left(\mathcal{E}^{l-1}\right)\right) \in \mathcal{E}^{k}, 1 \leq k \leq L_{j}, \\
& e^{k} \equiv \mathcal{E}^{k}\left(\mathcal{F}\left(e^{k-1}\right) ; \delta^{k}\right), 1 \leq k \leq n
\end{aligned}
$$

In the above equation, $\otimes$ indicates the convolution operator, $h()$ indicates the activation function, $\mathcal{F}$ () indicates down sample pooling as pooling helps in handling the global feature $\delta^{k}$ indicates parameter set of kth layer. Moreover, translation variance from pooling helps in reducing the number of parameter. Further PReLU is employed for activation and given in the next section.

\subsubsection{Activation through PReLu}

An activation function can be defined as,

$$
h(v)=\left\{\begin{array}{c}
v \text { if } v \text { is greater than zero } \\
\text { Av if } v \text { is less than or equal to zero }
\end{array}\right.
$$

$v$ indicates non-linear activation input, $h$ is non-linear activation, further to control coefficient of negative slope , A is used as the coefficient controlling; I indicates the variation of non-linear activation on the various channels. Moreover, in case if $\mathrm{A}$ is zero then it acts as ReLU, however when $\mathrm{A}$ is learnable parameter then it acts as PReLU (Parametric ReLU). We formulate the PReLU equation through below.

$$
h(v) \equiv \mathrm{A} \min (0, v)+\max (v, 0)
$$

A Is parameter which range between 0 and 1 ; final output $(\mathcal{K} ; \delta)$ of given feed forward is given as,

$(\mathcal{K} ; \delta)=\tilde{e}^{2 k-1}\left(\begin{array}{c}\cdots e^{k+1}\left(e^{k}\left(\cdots e^{2}\left(J ; \delta^{1}\right) ; \delta^{2}\right) \cdots ; \delta^{2}\right) ; \\ \left.\delta^{n+1}\right) \cdots ; \delta^{2 n-1}\end{array}\right)$ 
$\mathcal{K}$ Indicates input signal and $\delta$ is parameter set, further we compute the decoding part and convolution output $\tilde{e}^{t}$ is given as in (9),

$$
\begin{aligned}
& \tilde{e}_{s}^{t}=h\left(d_{t}^{s}+v_{t}^{s} \otimes \mathcal{W}\left(\tilde{e}^{s-1}\right)\right) \in \mathcal{E}^{s}, 1 \leq t \leq \mathcal{O}_{s} \\
& \tilde{e}^{t}=\tilde{\mathcal{E}}^{t}\left(v_{t}^{s} \otimes \mathcal{W}\left(\tilde{e}^{s-1}\right) ; \delta^{n+1}\right), n+1 \leq t \leq 2 n-1
\end{aligned}
$$

In the above equation, $\mathcal{W}()$ indicates up pooling which recovers the original matrix size, further convolution kernel is used for decoding and bias value is used for decoding in rth layer, also $\tilde{e}^{s-1}$ and $e^{s-1}$ is equal if $\mathrm{t}=\mathrm{n}+1$.

\subsection{U-Net}

A simple U-net architecture is shown in figure, where the intermediate level U-Net is applied for low resolution image. In here given input image $h$ is first filtered using the local filter $\Xi$, later it is optimized using the pooling operation $\Phi$, further this can be formulated through:

$$
\mathcal{E}=\phi^{P O}(h \circledast \Xi)
$$

$(h \circledast \Xi)$ Indicates filtering, $\phi^{P O}$ indicates pooling operator. Further since U-Net possesses by-pass connection; convolution coefficient is given through the below.

$$
W=M(h \circledast \Xi)
$$

Where $\mathbf{M}$ is computed as,

$$
\begin{aligned}
& M=\left[\mathcal{J} \phi \phi^{P O}\right] \\
& M^{\text {Trans }} M=\mathcal{J}+\phi^{P O}
\end{aligned}
$$
formulated as $\mathrm{v}$,

$\phi \phi^{P O}$ indicates matrix of rank deficient, considering the average pooling, these matrix are

$$
\begin{aligned}
& \left.U=\left[h \circledast \Xi \phi \phi^{P O} h \circledast \Xi\right)\right] \\
& \check{h}=b^{-1} \sum_{j=1}^{p}\left(h \circledast \Xi_{j} \circledast \Gamma_{j}+\phi \phi^{P O}\left(h \circledast \Xi_{i}\right) \circledast \delta_{j}\right)
\end{aligned}
$$

\subsubsection{Improvised U-Net}

U-Net is one of the popular base network, used for segmentation in bio medical image segmentation, however it has problem of low resolution as low resolution might cause smoothing of object boundaries, hence to avoid that we employ high level feature and low level feature. In here we adopt the U net and improvised in Figure 2 to achieve absolute ROI, further to achieve that proposed methodology uses two novel feature map, one is for High Level feature and another is for low level feature.

Considering the drawbacks of normal U-Net we have designed Improvised U-Net architecture, Improvised U-Net maintains the huge receptive field using the tight wavelets, i.e. here non-local transform $\phi^{P O}$ is applied instead of general pooling operation. Moreover the filter bank is given through the below.

$$
\phi=\left[\mathcal{R}_{1} \ldots \ldots \ldots \ldots \mathcal{R}_{m}\right]
$$

The below equation shows the tight filter bank where $R_{n}$ indicates sub-bank operator for given scalar c which is greater than zero.

$$
\phi \phi^{P O}=\sum_{n=1}^{m} \mathcal{R}_{n}^{T} \mathcal{R}_{n}=\mathcal{E} \mathcal{J}
$$




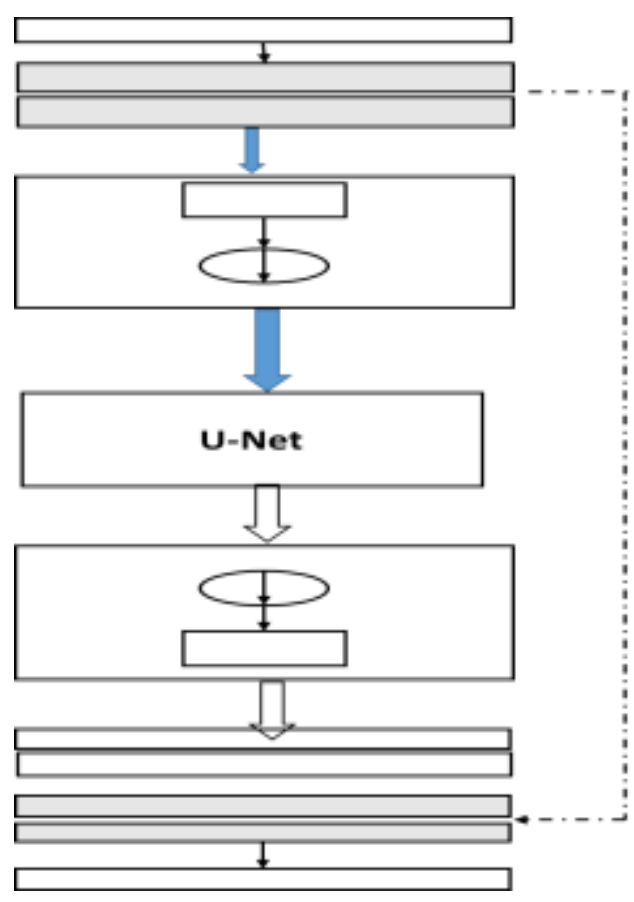

Figure 2. Improvised U-Net

Further convolution coefficient is written by:

$$
\mathcal{W}=Z(h \circledast \Xi)
$$

Where,

$$
\begin{aligned}
& Z=\left[\mathcal{J} R_{1} \ldots \ldots . R_{n}\right]^{T} \\
& \mathcal{E}=h \circledast \Xi \\
& \mathcal{Q}_{n}=C \mathcal{R}_{n}^{T} \\
& \mathcal{J}=\mathcal{R}_{n} \mathcal{R}_{n}^{T}
\end{aligned}
$$

Improvised U-Nets uses residual path for avoiding the low resolution information, however in improvised U-Nets places its residual path after pooling, this helps in achieving the high resolution information. Further to achieve we use adaptive filter, permeation rate is defined is given by:

$$
\mathrm{PR}=\left\{\begin{array}{c}
-0.5, \text { if } \mathrm{NM}_{y}(U, \mathrm{~A})<0.01 \\
\frac{\Sigma_{u, \mathrm{~A} \in \mathrm{obj}} \mathrm{l}(U, \mathrm{~A}) \mathrm{NM}_{y}(U, \mathrm{~A})}{\Sigma_{U, \mathrm{~A} \in \mathrm{obj}} \operatorname{label}(U, \mathrm{~A}) \mathrm{NM}_{x}(U, \mathrm{~A})}, \text { otherwise }
\end{array}\right.
$$

In (22), $\mathrm{NM}_{x}$ indicates normalized feature map after the residual path and $\mathrm{NM}_{y}$ indicates feature map after residual path; label indicates the object binary mask. In improvised U-Net, up sampling is carried by the de-convolution layer in residual operation and residual path at skip connection easily filters the size based information which is given by:

$$
\begin{aligned}
& e_{s, t}^{n+1}=h\left(y_{s, t}^{k+1} \otimes \mathrm{S}^{\mathcal{F}}\left(e^{k-1}\right)+c_{s, t}^{k+1}\right) \in e_{s}^{k+1} \\
& \mathcal{E}_{s, t}^{n+1}=\check{\mathcal{E}}_{s}^{k+1}\left(\mathcal{F}\left(e^{k-1}\right) ; \delta_{s}^{k+1}\right) \\
& e_{q, r}^{n+1}=h\left(y_{q, r}^{k+1} \otimes\left(e^{k-1}-e_{s}^{k+1}\right)+c_{q, r}^{k+1}\right) \in e_{q}^{k+1}, \\
& e_{s, t}^{n+1} \equiv \varepsilon_{q}^{k+1}\left(e^{k-1}-e_{v}^{k+1} ; \delta_{q}^{k+1}\right),
\end{aligned}
$$


$\varepsilon_{q}^{k+1}$ indicates object dependent up sampling, $\varepsilon_{r}^{k+1}$ indicates network output. Meanwhile above equation provides the output after the skip connection respectively. Further $\delta_{q}^{k+1}$ indicates parameter set, $c_{s, t}^{k+1}$ indicates bias value of vth kernel, $y_{q, r}^{k+1}$ indicates weight of vth kernel. Similarly, the above parameter is used in other equation for 1 th kernel and $\mathrm{r}$ bias value.

\subsection{High level and low level feature map}

Moreover feature map for High level are generated after the residual pass and extracts the given edge information for high-level feature, further this is formulated in the below. $r_{I U N}^{n+1}$ is proposed image before the convolution of layer $\mathrm{k}+1$, this is given in the below.

$$
r_{I U N}^{n+1}=\mathcal{W}\left(\mathcal{E}^{n}\left(\mathcal{F}\left(d^{k-1}\right) ; \delta^{k}\right)\right) \oplus \mathcal{E}_{u}^{n+1}\left(e^{n-1}-e_{s}^{n-1} ; \delta_{u}^{n+1}\right)
$$

High-level feature map is given through below,

$$
r_{I U N}^{n+1}=\mathcal{W}\left(\mathcal{E}^{n}\left(\mathcal{F}\left(d^{k-1}\right) ; \delta^{k}\right)\right) \oplus \mathcal{E}_{u}^{n+1}\left(e^{n-1}-e_{\text {low }}^{n-1} ; \delta_{u}^{n+1}\right)
$$

where $e_{v}^{n+1} \equiv e_{\text {low }}^{n-1}$

For the low level feature, feature map do not have problem of resolution issue and can used for global feature extraction, IUN indicates Improvised U-Net.

$$
\begin{aligned}
& r_{I U N}^{n+1}=\mathcal{W}\left(\mathcal{E}^{n}\left(\mathcal{F}\left(d^{n-1}\right) ; \delta^{k}\right)\right) \oplus \mathcal{E}_{t}^{n+1}\left(e^{n-1} ; \delta_{t}^{n+1}\right) \\
& \text { where } e_{u}^{k+1}=0
\end{aligned}
$$

Further these both feature are combined to achieve the better probable detection of Region of interest. Moreover in the next section Improvised U-Net is evaluated. In any deep learning-Neural network, there are high probability of overfitting a training dataset, hence we use dropout which is given in next section.

\subsection{Optimization through dropout}

In this section we tend to optimize the loss function, which avoids the overfitting; Moreover dropout is the particular regularization method which approximates huge dataset with various architecture parallel. Furthermore in optimization process several number of layers are dropped out i.e. it is temporarily removed with its all outgoing and incoming connections.

Moreover considering the above hidden layer denoted by $\mathcal{N}$ and index of hidden layer denoted by $n \in\{1, \ldots \ldots, \mathcal{N}\}$. In here $\mathcal{A}^{(n)}$ and $\mathcal{B}^{(n)}$ indicates input and output respectively. $\mathcal{V}^{(n)}$ indicates the weights of layer $\mathrm{n}$ and $\boldsymbol{b}(n)$ indicates biases at layer $\mathrm{n}$.

$$
\begin{aligned}
& a_{p}^{(n+1)}=d_{p}^{n+1}+\mathcal{V}_{p}^{(n+1)} b^{l} \\
& v_{p}^{(n+1)}=h\left(a_{p}^{n+1}\right)
\end{aligned}
$$

Hence, the feed forward of network with dropout is described by:

$$
\begin{aligned}
& t_{i}^{(n+1)} \sim g \\
& \ddot{b}^{(n)}=v^{(l)} * t^{(n)} \\
& a_{p}^{(n+1)}=b_{i}^{l+1}+\mathcal{V}_{i}^{(n+1)} \breve{a}^{(n)} \\
& b_{i}^{(l+1)}=h\left(a_{p}^{(n+1)}\right)
\end{aligned}
$$

In collective equation, $t^{(n)}$ indicates independent random variable of bernouli, $\breve{b}^{(n)}$ indicates thinned output created through $y^{(l)}$. These thinned output is used as the intermediate layer of input and it is applied to each layer for the larger network. Moreover through above process network is thinned or capacity is reduced, which avoids the overfitting Further derivatives of backpropagation is applied to this network for better learning. Once the model is trained through improvised U-Net and optimized through dropout layer, proposed model is evaluated considering various metrics and it is carried out in next section of this research work. 


\section{PERFORMANCE EVALUATION}

In this section we evaluate Improvised U-Net method, further in order to evaluate we have considered the ideal system configuration of windows 10 operating system packed with 8GB RAM, 2GB NVidia graphics with 1TB of Hard disk. Moreover, MATLAB is used as programming language with 2016b version.

\subsection{Dataset description}

In any machine learning based algorithm dataset plays a major role as data require for model training needs to be sufficient enough to perform the model. In here, we have used standard dataset from the open access Digital Database of thyroid ultrasound images from the Universidad Nacional de Colombia Laboratory [25]. Further, this dataset is utilized for computation of proposed model. Moreover, dataset comprises 92 images of thyroid ultrasound, out of theses 42 were from female and 50 from male from different age group. Moreover, these images were captured through TOSHIBA linear transducer and extracted from ultrasound video sequence. These thyroid nodules images are stored in ultrasonography model, which includes whole diagnostic description and annotation of thyroid lesions. Further, these are carried out under supervision of two radiologist expert using the TI-RADS lexicon. Proposed Improvised UNet is evaluated using various performance metric such as accuracy, F1-score, Dice coefficient and true positive. Dice coefficient depicts the similarity measure between the ground truth and area predicted, these metrics range from 0 to 1 . The more the value is closure to 1 , the better the model efficiency.

\subsection{Roi comparison}

Various methodologies proposed in [25-28] are used for comparative analysis. In this section, comparative analysis is carried out based on the ROI which is shown in Table 1, further the comparison is shown considering two images Table 1 comprises three column, first column shows the original image given in dataset, second column shows the ground truth of original image, third column is ROI achieved through the existing model [28] and fourth column shows the ROI achieved for Improvised U-Net model. Further through the Table 1 we observed there is huge difference in the ROI structure of existing model and ground truth, this is occurred due to the image quality used in the segmentation process of existing model whereas our model is nearer to the ground truth.

Table 1. comparison of ROI with existing methodology along with ground truth

Original image




\subsection{True positive}

A true positive is defined as an outcome where the proposed model predicts the positive class of particular model. Precisely, True positive is calculated as the ratio of positive prediction to the total number of positive. Furthermore Figure 3 presents the comparison of various methodology with proposed model and comparative analysis shows that [26] achieves only $52.26 \%$ of true positive rate, although [27] and NDRLS [25] achieves 93.51 and 95.44 respectively, still it underperforms. Further, existing $96.44 \%$ of true positive rate whereas Improvised U-Net achieves nearly absolute true positive rate of $98.95 \%$.

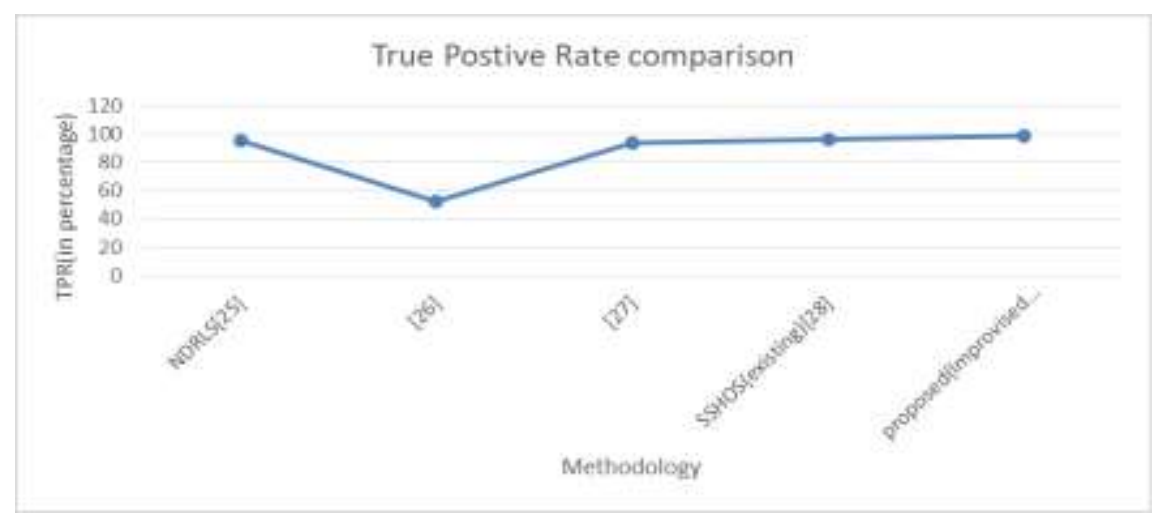

Figure 3. Comparison of various methodology considering true positive rate

\subsection{Dice coefficient}

In segmentation, Dice Coefficient is an evaluation metric which is used for gauging the similarity between two given image. In general Dice coefficient is ratio of $2 *$ AoO (Area of overlap) to total number of pixels in given images. Figure 4 shows the comparison of various existing model with proposed Improvised U-Net model, in here [26] achieves least dice coefficient pf 67.93, [27] achieves 80.85, existing model SSHOS achieves 92.24, NDRLS achieves 94.2 whereas proposed model achieves 95.60.

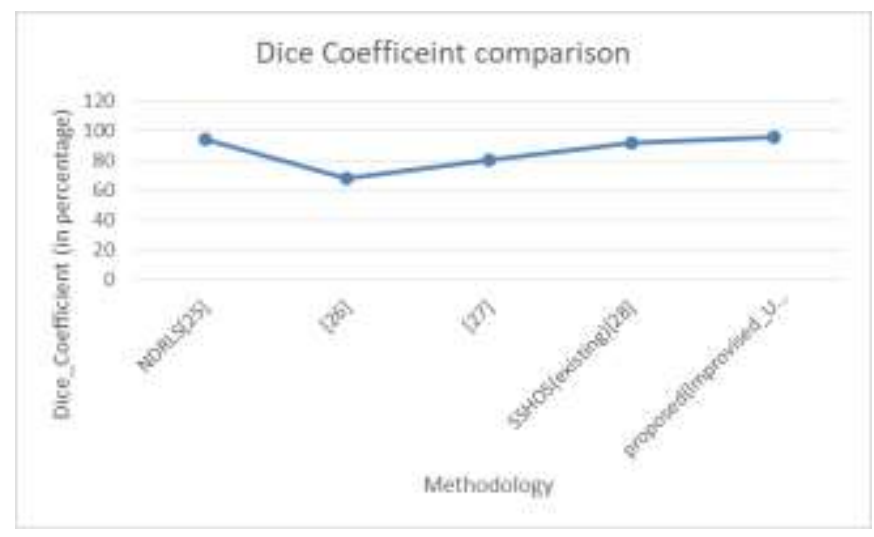

Figure 4. Comparison of various methodology considering dice coefficient metrics

\subsection{Accuracy, F1-measure and specificity}

Accuracy is one of the important performance metric measure in classification process; accuracy presents the correctly predicted model, further it is defined as ratio of number of sample correctly predicted to the total number of samples. Moreover this can be formulated through the below.

$$
\operatorname{accuracy}=\frac{C P}{T S}
$$


Where CP the number of is correct prediction and TS is total number of samples. Evaluation of various metrics is tabulated in Table 2. Our model achieves the accuracy of $98.39 \%$.

F1 score is measurement of test's accuracy; further F1 measure is computed using precision and recall. In general, we can say that F1 measure is the harmony mean of precision and recall. Improvised U-Net achieve F1-score of 91.87. Specificity which is also known as TNR (true negative rate) is measure of given portion of absolute negatives which are correctly classified, it is one of the highly appreciated and popular metrics in biomedical field. Higher value of specificity indicates better efficiency of model, in here. Through the comparative analysis it is observed that our model not only performs better in terms of comparative analysis but also considers the various parameter which is mostly ignored by other segmentation methodology.

Table 2. Evaluation of various metrics

\begin{tabular}{cc}
\hline Performance_Metrics & Value observed (in percentage) \\
\hline Accuracy & 98.395 \\
F1-Meaure & 0.918762 \\
Specificity & 0.98744 \\
\hline
\end{tabular}

\section{CONCLUSION}

Thyroid segmentation is one of the essential precursor for several quantitative analysis for thyroid disease. However, segmentation is major challenging considering the existence of similar structure such as lymph node, ambiguous boundary, low contrast and signal to noise ratio. Further, it has inhomogeneous appearance this makes more difficult for segmentation and thus it is classified as patch classification task. In this paper considering the U-Net advantage, we develop an improvised U-Net for segmentation of Ultrasound image, in here we develop two feature map namely high level feature and low level feature for better resolution and better information regarding the image; further we deploy dropout layer for optimizing the performance and achieve better metrics. Improvised U-Net is evaluated on standard dataset of DDTI considering performance metrics like true positive, specificity, F1-measure and accuracy through the performance analysis it is observed that improvised U-Net achieves accuracy of 98.39\%, F1-measure of 91.87, Dice Co-efficient of 95.60 and true positive rate which is better than any existing model till the date this research is carried out. Although our proposed model achieves better in terms of defined metrics, still there are several area such as considering further large dataset and other metrics.

\section{REFERENCE}

[1] K. Bibbins-Domingo, "Screening for thyroid cancer: US preventive services task force recommendation statement," Jama, vol. 317, no. 18, pp. 1882-1887, 2017.

[2] Bikas and K. D. Burman, "Epidemiology of thyroid cancer," in The Thyroid and Its Diseases, M. Luster, L. Duntas, and L. Wartofsky, Eds. Basel, Switzerland: Springer, pp. 541-547, 2019.

[3] Smriti Sahu, Maheedhar Dubey, Mohammad Imroze Khan, "Comparative Analysis of Image Enhancement Techniques for Ultrasound Liver Image," International Journal of Electrical and Computer Engineering (IJECE), vol. 2, no. 6, pp. 792 797 ISSN: 2088-8708, 2012.

[4] V. Kumar, J. M. Webb, A. Gregory, M. Denis, D. D. Meixner, M. Bayat, D. H. Whaley, M. Fatemi, and A. Alizad, "Automated and real-time segmentation of suspicious breast masses using convolutional neural network," PLoS ONE, vol. 13, no. 5, 2018, Art. no. e0195816.

[5] F. Milletari, S.-A. Ahmadi, C. Kroll, A. Plate, V. Rozanski, J. Maiostre, J. Levin, O. Dietrich, B. Ertl-Wagner, K. Bötzel, and N. Navab, "Hough- CNN: Deep learning for segmentation of deep brain regions in MRI and ultrasound," Comput. Vis. Image Understand., vol. 164, pp. 92-102, 2017.

[6] J. Yang, L. Tong, M. Faraji, and A. Basu, "IVUS-net: An intravascular ultrasound segmentation network," 2018, arXiv:1806.03583. [Online]. Available: http://arxiv.org/abs/1806.03583

[7] C. Azzopardi, Y. A. Hicks, and K. P. Camilleri, "Automatic carotid ultrasound segmentation using deep convolutional neural networks and phase congruency maps," in Proc. IEEE 14th Int. Symp. Biomed. Imag. (ISBI), pp. 624-628, 2017.

[8] Koundal D, Gupta S, Singh S, "Automated delineation of thyroid nodules in ultrasound images using spatial neutrosophic clustering and level set," Appl Soft Comput, vol. 40, pp. 86-97, 2016.

[9] SavelonasMA, Iakovidis DK, Legakis I,Maroulis D, "Active contours guided by echogenicity and texture for delineation of thyroid nodules in ultrasound images," IEEE Trans Inf Technol Biomed, vol. 13, no. 4, pp. 519-527, 2009.

[10] Bhargav Bhatkalkar, Abhishek Joshi, Srikanth Prabhu, Sulatha Bhandary, "Automated fundus image quality assessment and segmentation of optic disc using convolutional neural networks," Home, vol. 10, no. 1, 2009.

[11] BrijeshN.Shah1, JayminK.Bhalani2, "Comparative analysis and implementationof structured edge active contour," International Journal of Electrical and Computer Engineering (IJECE), vol. 10, no. 2, pp. 1842 1848, 2020, ISSN: 2088-8708, DOI: 10.11591/ijece.v10i2.pp1842-1848. 
[12] C. Li , C. Xu , C. Gui , M.D. Fox, "Distance regularized level set evolution and its application to image segmentation," IEEE Trans. Image Process, vol. 19, no. 12, pp. 3243-3254, 2010.

[13] [C. Azzopardi, Y. A. Hicks, and K. P. Camilleri, "Automatic carotid ultrasound segmentation using deep convolutional neural networks and phase congruency maps," in Proc. IEEE 14th Int. Symp. Biomed. Imag. (ISBI), pp. 624-628, 2017.

[14] C. Li, C. Xu, C. Gui, and M. D. Fox, "Distance regularized level set evolution and its application to image segmentation," IEEE Trans. Image Process., vol. 19, no. 12, pp. 3243-3254, 2010.

[15] Dewi Putrie Lestari, Sarifuddin Madenda, Ernastuti, Eri Prasetyo Wibowo, "Comparison of Three Segmentation Methods for BreastUltrasound Images Based on Level Setand Morphological Operations," International Journalof Electrical and Computer Engineering (IJECE), vol. 7, no. 1, pp. 383-391, 2017. ISSN: 2088-8708, DOI: 10.11591/ijece.v7i1.pp383-391.

[16] C.-Y. Chang, Y.-F. Lei, C.-H. Tseng, and S.-R. Shih, "Thyroid segmentation and volume estimation in ultrasound images," IEEE Trans. Biomed. Eng., vol. 57, no. 6, pp. 1348-1357, 2010.

[17] Moh'dRasoulAl-Hadidi1, Bayan Al Saaidah2, Mohamme dY.Al-Gawagzeh, "Glioblastomasbrain tumoursegmentation based on convolutional neural networks," International Journal of Electrical and Computer Engineering (IJECE), vol. 10, no. 5, pp. 4738-4744, 2020. ISSN: 2088-8708, DOI: 10.11591/ijece.v10i5.pp47384744.

[18] M. Freesmeyer, L. Knichel, C. Kuehnel, and T. Winkens, "Stitching of sensor-navigated 3D ultrasound datasets for the determination of large thyroid, volumes-a phantom study," Med. Ultrasonography, vol. 20, no. 4, pp. 480-486, 2018.

[19] T. Wunderling, B. Golla, P. Poudel, C. Arens, M. Friebe, and C. Hansen, "Comparison of thyroid segmentation techniques for 3D ultrasound," Proc. SPIE Int. Soc. Opt. Photon., vol. 10133, 2017, Art. no. 1013317.

[20] C.-Y. Chang, Y.-F. Lei, C.-H. Tseng, S. R. Shih, "Thyroid segmentation and vol- ume estimation in ultrasound images," IEEE Trans. Biomed. Eng. vol. 57, no. 6, pp. 1348-1357, 2010.

[21] T. Loupas, W.N. McDicken, P.L. Allan, "An adaptive weighted median filter for speckle suppression in medical ultrasonic images," IEEE Trans. Circuit Syst. vol. 36, no. 1, pp. 129-135, 1989.

[22] D. Selvathi, V. S. Sharnitha, "Thyroid classification and segmentation in ultra- sound images using machine learning algorithms," in: Proceedings of Interna- tional Conference on Signal Processing, Communication, Computing and Net- working Technologies, pp. 836-841, 2011.

[23] H. Garg, A. Jindal, "Segmentation of thyroid gland in ultrasound image using neural network", in: Proceedings of International Conference on Computing, Communications and Networking Technologies, pp. 1-5, 2013.

[24] P. Poudel, A. Illanes, C. Hansen, M. Friebe, "Evaluation of commonly used al- gorithms for thyroid ultrasound images segmentation and improvement using machine learning approaches," J. Healthcare Eng. 2018, pp. 1-13, 2018.

[25] S. Poonguzhali andG. Ravindran, "Acomplete automatic region growing method for segmentation of masses on ultrasound images," in Proceedings of the 2006 International Conference on Biomedical and Pharmaceutical Engineering, ICBPE 2006, pp. 88-92, 2006.

[26] M. M. Abdelsamea, G. Gnecco, and M. M. Gaber, "A SOMbased Chan-Vesemodel for unsupervised image segmentation,” Soft Computing, vol. 21, no. 8, pp. 2047-2067, 2017.

[27] D. Koundal, S. Gupta, and S. Singh, "Computer aided thyroid nodule detection system using medical ultrasound images,” Biomedical Signal Processing and Control, vol. 40, pp. 117-130, 2018.

[28] Haji, Salih \& Yousif, Raghad. (2019). A Novel Neutrosophic Method for Automatic Seed Point Selection in Thyroid Nodule Images. BioMed Research International, pp. 1-14, 2019. 10.1155/2019/7632308. 